Vers une approche communicationnelle de l'individu face aux épreuves organisationnelles

A Communicational Approach to the Individual in the Face of Organizational Challenges

François Lambotte

\title{
OpenEdition
}

Journals

Édition électronique

URL : http://journals.openedition.org/edc/7454

DOI : 10.4000/edc.7454

ISSN : 2101-0366

Éditeur

Université de Lille

Édition imprimée

Date de publication : 1 juin 2018

Pagination : 33-56

ISBN : 978-2-917562-19-2

ISSN : $1270-6841$

Référence électronique

François Lambotte, «Vers une approche communicationnelle de l'individu face aux épreuves organisationnelles », Études de communication [En ligne], 50 | 2018, mis en ligne le 01 juin 2020, consulté le 04 janvier 2020. URL : http://journals.openedition.org/edc/7454 ; DOI : 10.4000/edc.7454

(c) Tous droits réservés 


\section{Vers une approche communicationnelle de l'individu face aux épreuves organisationnelles}

A Communicational Approach

to the Individual in the Face of Organizational Challenges 
Résumé / Abstract

Nous inscrivant dans une démarche inductive, nous avons procédé à une lecture systématique des dossiers thématiques de la revue Études de communication dédiés au couple communication et organisation. D'un point de vue phénoménologique, cette lecture a mis en lumière la fragmentation de l'organisation et la montée en puissance du questionnement sur l'individu. Alors que des auteurs comme Martuccelli considèrent que la sociologie classique n'est pas armée pour traiter de cette réalité sociale, nous proposons de faire ressortir des pistes de recherches et de développement futures pour une approche communicationnelle de la constitution des individus.

Mots-clés : individualisation, épreuves organisationnelles, constitution des individus.
Using an inductive approach, we conducted systematic analysis of the five thematic issues of Études de communication devoted to the question of organizational communication. From a phenomenological point of view, this analysis highlights the fragmentation of the organization and the emergence of questions concerning the individual. While authors such as Martuccelli consider that classical sociology is not able to deal with this social reality, we point out some future avenues for the development of a communicational approach to the constitution of individuals.

Keywords: individualization, organizational challenge, constitution of individuals. 


\section{Introduction}

Le couple communication et organisation a fait l'objet de cinq numéros thématiques de la revue scientifique Études de communication. La lecture et l'analyse transversale de ces cinq dossiers complets offre une perspective intertextuelle propice à l'identification de marqueurs phénoménologiques et théoriques dans le traitement communicationnel de phénomènes organisationnels. D'un point de vue phénoménologique, ce travail met en lumière la montée en puissance du questionnement sur l'individu au regard de l'organisation.

Pour Martuccelli, cela témoigne de ce que les sociologues appellent « la fin d'une idée de totalité sociétale analytiquement harmonieuse » $(2009,19)$. Mettre l'individu au centre de la réflexion part de la nécessité « de reconnaître la singularisation croissante des trajectoires des individus et ce même lorsqu'ils occupent des positions sociales similaires » $(2009,19)$. Là, où hier les normes semblaient être reproduites de façon « plus ou moins homogènes par les institutions, elles semblent aujourd'hui être engendrées in situ et de manière purement réflexive par les acteurs » $(2009,20)$.

Nous détaillerons, dans un premier temps, la manière dont les auteurs des 38 articles analysés traitent de l'individu aux prises avec l'organisation. Nous verrons que les travaux repris dans les cinq dossiers thématiques reposent sur ce que Martuccelli nomme deux stratégies intellectuelles de l'individualisation, à savoir la socialisation et la subjectivation. Cependant plusieurs sociologues (e.a., Alain Touraine et Danilo Martuccelli) considèrent qu'une troisième stratégie centrée sur l'individu est envisageable, à savoir l'individuation. L'individu devient, dans cette troisième voie, le pivot de la réflexion et il faut en conséquence accorder un rôle analytique à l'individualisation " pour décrire le processus de différenciation croissante des parcours personnels " (Martuccelli, 2009, 22). Dès lors, nous questionnerons, dans un second temps, les approches communicationnelles sur lesquelles reposent les articles des cinq numéros thématiques au regard de l'agenda de recherche proposé par Danilo Martuccelli afin de faire ressortir des pistes de recherches et de développements pour une approche communicationnelle de la constitution des individus.

\section{1. Méthodologie}

Nous inscrivant dans une démarche inductive (Strauss et Corbin, 1998), nous avons procédé à une lecture systématique d'un corpus constitué de 38 articles scientifiques. Ce corpus n'inclut pas les introductions rédigées par les éditeurs.trices (Noyer et Chantraine, 2001 ; Heller, 2005b ; Guyot, 2009a ; de la Broise et Grosjean, 2010 ; Morillon et al., 2013), souhaitant nous faire notre propre opinion sur le fil rouge qui se dégage des propositions constituant chaque dossier. La sélection de ces dossiers thématiques reprend les dossiers 
dont la thématique met explicitement en question l'organisation. Ainsi, elle ne porte pas sur d'autres articles, analysés par Pierre Delcambre, qui traitent notamment des écrits au travail dont l'apport aux SIC et à la communication organisationnelle n'est plus à prouver. De même la sélection ne reprend pas les travaux qui précèdent la création officielle de la revue Études de Communication, ni ceux qui, en varia, traitent de la communication organisationnelle. En ce sens, cette analyse présente certaines limites dont il faut tenir compte. Cela étant, le choix des dossiers thématiques permet d'analyser les articulations entre les articles traitant d'une problématique centrale au projet éditorial.

Nous avons procédé au codage des articles par le biais d'annotations et d'inventaires de caractéristiques " classiques " d'un article scientifique en sciences sociales :

- L'objectif de l'article tel qu'il est déclaré par les auteurs, eux-mêmes. L'objectif se constitue généralement de l'objet ou du phénomène étudié mais aussi de l'angle d'attaque proposé par les auteurs. Par exemple, "Nous proposons dans cet article de relire différents travaux sur les centres d'appel en questionnant la mise en jeu du corps dans l'activité de téléphonie. Le choix de ce regard s'appuie sur l'hypothèse qu'il existe un lien entre l'activité d'information et la posture corporelle » (Kogan, 2009, 243).

- Le paradigme dans lequel s'inscrit chaque article, à savoir les hypothèses méta-théoriques sur la nature des sujets étudiés ici la communication et l'organisation qui ont présidées à la démarche des auteurs. Nous avons déduit le paradigme de notre lecture de l'article et appliqué la catégorisation proposée par Giroux et Demers (1998) : paradigme critique (14 articles sur 38), interprétatif (13) et constructionniste (11).

- L'appareillage conceptuel et théorique développé ou sur lequel repose la recherche ou la démonstration.

- Le dispositif méthodologique envisagé au regard des concepts ou du cadre théorique en lien avec l'existence et le traitement d'un corpus empirique pour appuyer le propos.

Une colonne " annotation et commentaires " nous a également aidé à tisser, au fur et à mesure de nos lectures, des liens entre les articles d'un numéro ou au travers des cinq numéros. Un tableau de synthèse en annexe 1 reprend les métadonnées des cinq dossiers analysés. Malgré la diversité des objets et des approches théoriques identifiées, ces travaux convergent quant à la place centrale donnée à l'individu dans la réflexion sur les phénomènes organisationnels étudiés. 


\section{2. \\ L'individu aux prises avec l'organisation}

En confrontant les travaux développés aux trois stratégies intellectuelles proposées par Martuccelli pour étudier l'individu, nous constatons que ceux-ci s'inscrivent essentiellement dans deux des trois voies stratégiques : la socialisation et la subjectivation.

\subsection{La voie de la socialisation}

"La socialisation désigne dans un seul et même mouvement le processus par lequel les individus, en acquérant les compétences nécessaires, s'intègrent à une société, et la manière dont une société se dote d'un type d'individu. Toutes les théories de la socialisation rendent donc compte de la tension entre les aspects naturels, les compétences innées, et les dimensions culturelles d'un acteur socialement constitué. Les individus se construisent, sinon toujours en reflet, au moins en étroite relation avec les structures sociales : valeurs d'une culture, normes de conduite, institutions, clivages sociaux, classes, styles familiaux » (Martuccelli, 2005, 2).

Dans une approche info-communicationnelle, les auteurs questionnent l'organisation par le biais des discours, des écrits, des projets, des systèmes d'information, des techniques ou des technologies, des dispositifs ou des procédures. Ces formes et normes (Le Moënne, 2013) ou ces figures organisationnelles (Cooren, 2010) se manifestent aux acteurs de l'organisation dans leurs pratiques professionnelles que ce soit dans la réalisation d'une activité quotidienne, dans la négociation ou la contribution aux projets organisationnels. L'organisation est pensée comme un ensemble fait de structures sociales (de techniques, de normes, de systèmes) en relation avec lesquelles les individus se construisent, s'adaptent ou encore s'opposent. Les extraits qui suivent illustrent la manière dont les articles analysés appréhendent cette socialisation de l'individu par le prisme de la communication.

Sur la base d'une analyse des usages par les parties prenantes d'une coopérative vinicole, André Salançon (2009) montre brillamment l'effet du système d'information sur les pratiques et sur la distribution du pouvoir

«En tout état de cause, la traçabilité des objets ne peut être dissociée d'une réelle interaction entre ce dispositif règlementaire et les relations entre les acteurs qui demeurent dynamisées par les stratégies permanentes de pouvoirs. Si l'information peut être considérée selon un certain point de vue comme un acteur à part entière, l'adoption et la maîtrise des technologies d'information créent un espace de médiations des pouvoirs que se disputent les acteurs déployant par ailleurs leur propre stratégie »(Salançon, 2009, 165). 
Les dispositifs deviennent aussi des lieux où se jouent et se rejouent les relations de pouvoir, parfois de façons conflictuelles (Gleonnec, 2009) entre les acteurs parfois de façons tactiques chacun tentant d'y trouver les leviers de déploiement de sa politique, les interstices ou les recoins permettant d'y échapper. Dans leur analyse du dispositif de déclarations des risques et des incidents en milieu hospitalier, Anne Mayère et Angélique Roux (2009) se penchent sur l'écrit au travail afin de comprendre pourquoi certaines choses sont éludées notamment afin de laisser des interstices permettant aux divers acteurs de la pratique d'écriture une expression ou interprétation plurielle dans leur pratique.

D'autres auteurs montrent que les acteurs organisationnels ne sont pas sans ressource ni dénués d'intelligence face aux épreuves organisationnelles. Isabelle Comtet (2009) rend compte de la marge de liberté "laissée " et les facultés d'adaptations des acteurs dans l'appropriation d'un dispositif sociotechnique dont la mise en œuvre s'est avérée efficace. Elle met en exergue que "les enjeux liés à la situation de travail (1) et les impératifs du fonctionnement organisationnel (2) poussent, implicitement les salariés à façonner par essais et erreurs les dispositifs techniques nécessaires à leur activité (3) " (Comtet, 2009, § 25).

\section{Dans la même veine, Maryse Carmes $(2009, \S 16)$ démontre}

«Qu'analyser les manières dont résonnent, de façon tantôt convergente et tantôt divergente, les politiques organisationnelles et leurs dispositifs idéels et matériels dédiés à la performation stratégique et tactique des processus de travail, ainsi que les micro-pratiques avec leurs propres narrations, permet d'observer la manière dont les salariés, en cherchant tout à la fois à adopter les nouveaux dispositifs, ne cessent aussi d'introduire altération et création vis-à-vis des scripts managériaux et techniques ».

Dans leur article, Daniel Robichaud et Chantal Benoit-Barné (2010) examinent les conversations des participants à un comité mettant sur pied un processus de consultation des membres de l'organisation. Dans ce cadre, les participants sont amenés à discuter la version préliminaire d'un texte énonçant les objectifs du comité qui sera présenté aux employés. La recherche détaille comment les normes d'écritures (l'écriture « technique et managériale » versus l'écriture "grand public ») peuvent s'opposer et être négociées par l'entremise des parties prenantes. Mais la négociation de ces normes d'écriture est également symboliquement forte puisqu'elle remet implicitement en cause le mandat du groupe et in fine questionne l'acceptation du plan stratégique dont ce comité est le reflet opérationnel.

Enfin, certains articles étudient les tensions entre les figures multiples ou en transformation de l'individu (travailleur, employé, citoyen, client) produites par le biais des processus de communication et de médiatisation de l'organisation. A titre d'exemple, l'article de Jacques Noyer (2001) se penche sur la production par le discours médiatique des figures de l'usager (TGV) et du 
service public. L'auteur explore les contrastes et les tensions entre les figures de l'usager et celles du service public (ici, le transport en commun et le TGV). Brigitte Guyot (2009b) examine, quant à elle, comment les dispositifs informationnels façonnent les rôles, les attentes et les capacités des acteurs. Elle invite le lecteur à se questionner sur le système d'information plus large que le système informatique pour analyser ce qu'il induit. La multiplication des outils oblige à créer des tactiques de sélection de l'information et des outils. Elle met également en évidence la tension entre produire de l'information pour soi et pour autrui : produire pour autrui induit un travail éditorial plus lourd alors que les exigences de productivité augmentent. Cela expliquerait en partie l'échec des outils collectifs de production d'information.

\subsection{La voie de la subjectivation}

Si les auteurs, ci-dessus, font la part belle à l'intelligence des acteurs et à ses capacités de socialisation, le paradoxe et les tensions entre individu(s) et projet organisationnel sont par ailleurs très présentes et fortement dénoncées dans les textes reposant sur un paradigme critique. Ceux-ci sont repris principalement dans les numéros 23 (Noyer et Chantraine, 2001) et 28 (Heller, 2005b).

Ces articles s'inscrivent dans une seconde stratégie intellectuelle de l'étude de l'individu : la subjectivation :

«L'étude de la subjectivation est indissociable de la conception d'une modernité soumise à l'expansion continue du processus de rationalisation, à savoir, l'expansion de la coordination, de la planification, de la prévision croissante dans toutes les sphères de la vie sociale (de l'économie au droit, de la politique à l'art comme l'a énoncé Weber). C'est sur l'arrière-plan de ce mouvement de contrôle social, que se pose la problématique fondamentale de la subjectivation : comment parvenir à imaginer la possibilité d'une émancipation humaine ? » (Martuccelli, $2005,4)$.

Plusieurs articles du numéro 23 questionnent la façon dont de nouvelles formes de management ou de contrôle s'imposent aux acteurs par le biais de techniques de management, de nouveaux équipements, de dispositifs d'information de leur activité. Par l'analyse des discours sur les TIC dans les administrations publiques, Bruno Raoul (2001) fait l'hypothèse que le discours déterministe participe à la montée en puissance du discours managérial dans les administrations. De son côté, Thomas Lamarche (2001) propose une réflexion économique et critique dénonçant la marchandisation du service public et la rationalisation du travail. Pour l'auteur, les différentes techniques et les discours sur le marketing, l'orientation client, la mise en concurrence, la responsabilisation ont pour effet de créer un mode de pensée marchand dans un monde de service public et civique. L'argument repose ici sur le fait que les techniques et les discours sur la modification des pratiques, l'individualisation du service et la logique marketing sont performatifs dans le sens où ils créent le statut 
lui-même de client et les attentes qui y sont associées.

Dans le numéro 28 , le projet éditorial est le plus serré théoriquement puisqu'il propose aux auteurs de se pencher sur les nouvelles formes d'assujettissements en organisation par le prisme Foucaldien. A travers une série d'essais théoriques, les auteurs démontrent par l'analyse critique du discours sur les nouvelles formes et techniques de management (e.a., flexibilité, responsabilisation des employés, management par objectifs, management par le projet, leadership, etc.) le glissement de l'exercice du pouvoir par le corps vers l'exercice du pouvoir par le psyché (De Gaulejac 2005 repris par Heller, 2005a, § 8) :

"C'est pour une bonne part, comme force productive que la psyché est investie de rapports de pouvoir et de domination. La psyché ne devient force utile que si elle est à la fois énergie productive et énergie assujettie. On peut reprendre presque mot pour mot l'analyse de Michel Foucault en substituant la psyché au corps comme objet du pouvoir dans les entreprises hypermodernes [...]. II ne s'agit plus de rendre les corps 'utiles et dociles', mais de canaliser le maximum d'énergie libidinale pour la transformer en énergie productive » (p. 84-85).

Ainsi Vincent Petitet (2005) explique qu'une nouvelle forme de gouvernementalité managériale, c'est-à-dire l'emprise du management sur nos manières de faire et de penser, se développe par le biais de modalités opératoires que sont $(2005, \S 19)$ :

- La gestion de la docilité qui est autant production d'une uniformisation que normalisation des comportements et des corps ;

- La généralisation d'un discours fictionnel qui prétend produire une vérité transcendant le présent de l'organisation ;

- L'appropriation stratégique de la communication qui fait de la production du sens et de la parole des moyens de pérenniser une économie de l'obéissance.

D'autres auteurs mettent en exergue également le paradoxe entre logique de libéralisation du travail et logique de rationalisation post-disciplinaire (Bouillon, 2005 ; Estienne, 2005). Face à ce paradoxe, on comprend le questionnement quant aux possibilités d'émancipation de l'individu. Ces deux passages repris du texte de Floris et Ledun (2005) synthétisent merveilleusement le propos de ce dossier à ce sujet : 
«Un double autocontrôle s'instaure : celui de chaque individu sur ses performances, et celui des équipes de travail sur chaque membre. Le modèle de l'autocontrôle (et la peur de perdre l'emploi) gagne sur deux tableaux : il court-circuite les tendances à former des contre-pouvoirs collectifs par l'individualisation des salariés mis en concurrence, et il déplace la responsabilité des dirigeants vers la pression incontestable de la 'demande' et de la concurrence » (Floris et Ledun, 2005, § 6).

Les deux auteurs ajoutent $(2005, \S 16)$ que :

«Dans les entreprises, Vincent De Gaulejac observe que le pouvoir disciplinaire faisait appel au Surmoi des individus, c'est-à-dire au respect psychiquement intériorisé de la Loi sociale et des 'grands Autres' qui l'instituent. Aujourd'hui, en même temps que l'entreprise est érigée en institution principale de référence (Enriquez), c'est le Moi narcissique, antérieur à la structuration œdipienne du Surmoi, qui est sollicité par le nouveau management au travers de la peur d'être exclu, de la honte de ne pas être à la hauteur, ou de n'être plus reconnu, ainsi que du désir de toute-puissance auto-réalisatrice. L'employabilité, la mobilité, la course à la performance et l'individualisation de la relation salariale sont les vecteurs de la sollicitation narcissique des individus au travail. La peur du chômage et de la précarité est l'autre face insécable de cette sollicitation. Les craintes archaïques enfouies dans le narcissisme primaire sont constamment excitées par la précarisation sociale et psychique des individus atomisés ".

Si les articles témoignent d'une importance accrue accordée à l'individu, ceux-ci semblent pour autant ancrés dans deux voies classiques que sont la socialisation et la subjectivation. Les travaux de Martuccelli, en particulier, appellent au développement d'une troisième voie - celle de l'individuation qui semble peu développée, à tout le moins dans les cinq dossiers analysés :

"Derrière la notion d'individuation, notamment en Allemagne et en Angleterre, et d'une manière un peu différente et moins consensuelle en France, se répand l'idée que dans la mesure où la société (ou les institutions) ne sont plus censées transmettre de manière harmonieuse des normes d'action, il revient aux individus de donner un sens, grâce notamment à l'expansion de la réflexivité, à leurs trajectoires (Beck, [1986], 2001 ; Giddens, 1991 ; Dubet, 1994 ; Bauman, 2001) » (Martuccelli, 2005, 8).

Dans la seconde partie de cet article, nous expliquons l'agenda de recherche proposé dans cette stratégie de l'individuation. Nous mettons enfin en perspective les approches théoriques qui sous-tendent les 38 articles analysés au regard de cet agenda. II ne s'agit pas de délaisser les deux premières voies mais d'envisager ce que pourrait apporter l'individuation à nos approches communicationnelles de l'organisation et de l'individu. 


\section{3. \\ Vers une approche communicationnelle de la constitution de l'individu?}

\subsection{Principes et agenda d'une sociologie de l'individuation}

L'agenda de recherche de cette stratégie de l'individuation repose sur une série de principe que nous synthétisons ici. L'un des premiers principes est de partir des individus. Celui-ci n'implique pas nécessairement de partir d'une approche interactionniste, nous dit Martuccelli, mais bien du fait « que l'individu est sollicité d'une manière de plus en plus singulière par un ensemble d'institutions sociales (éducation, droits, opportunité d'emploi, processus de mobilité sociale...), l'enjoignant de développer une biographie personnelle de plus en plus singulière - ce que souligne d'ailleurs justement la notion d'individualisation » $(2005,9)$.

Ces travaux se caractérisent également par une description très fine des échanges au plus près des acteurs. L'expression des sentiments mais également le travail sur soi y occupent également une place importante. On s'attardera dès lors à étudier les raisons et les intentions de l'agir en relation avec la construction de l'individu.

Cela étant, même si l'individu est le point d'entrée sur le terrain, « au point d'arrivée : une fois l'excursus analytique accompli, ce sont bien les principales manières possibles dont on peut être un individu qui ont été décantées " (Martuccelli, 2005, 9). L'objet n'est pas l'individu mais bien les enjeux sociétaux auxquels l'individu est confronté et qui prennent la forme d'épreuves sociétales dont l'intelligibilité sera construite par le prisme de l'individu.

Pour y arriver, Martuccelli (2009) propose une stratégie d'analyse centrée sur l'individuation qui désigne un processus à la fois diachronique et synchronique qui interroge les grandes transformations historiques d'une société à l'échelle de l'individu. Les parcours d'individuation y sont envisagés sur un temps long (à l'échelle d'une génération) confrontés à des épreuves et ce afin de questionner par ce biais la manière dont les individus sont produits et se produisent.

Les épreuves telles que définies par Martuccelli ont quatre grandes caractéristiques. D'abord, la notion d'épreuve est indissociable de celle du récit qui permet de mettre en exergue la tension, l'ambivalence, les paradoxes entre lesquels les individus sont contraints de naviguer. Ensuite, ce récit doit laisser de la place à la subjectivité, au ressenti personnel qui met en évidence l'effort déployé pour faire face à l'épreuve. L'épreuve, en troisième lieu, est associée aux notions d'évaluation, d'apprentissage et de sélection. Quel est l'apprentissage de l'individu à l'issue de l'épreuve, que décide-t-il de faire à l'issue de celle-ci ? Quatrièmement, les épreuves se doivent d'être le reflet des grands enjeux sociétaux. Une première série d'épreuves proposées sont l'école, le 
travail, la ville et la famille. Une seconde série renvoie à différentes dimensions du lien social : rapport à I'histoire, aux collectifs, aux autres, à soi-même. Le centre de gravité n'est néanmoins pas l'épreuve en tant que telle mais " bien l'ensemble des épreuves auxquelles un individu est socialement confronté » (Martuccelli, 2009, 25). Les épreuves se succèdent, se chevauchent tout au long de la vie de chaque individu. C'est dans la comparaison des portraits que I'on peut rendre compte de l'articulation entre les transformations structurelles (vues comme des épreuves) et les jeux de places tels qu'en témoignent les récits des individus.

Ces récits de vie servent enfin à dresser des portraits qui se distinguent par leur singularité. II s'agit en effet d'éviter de faire du portrait une illustration d'un phénomène social. Le portrait implique au contraire de prêter une attention particulière à l'intelligence de l'individu, qu'elle soit rationnelle ou subjective. Le portrait doit aussi mettre en exergue le travail sur soi que chaque acteur " accomplit afin de se fabriquer en tant que sujet... à interroger de près les variations intra- et interindividuelles, à interroger comment et pourquoi certains y parviennent et d'autres pas " (Martuccelli, 2009, 27).

3.2. Cinq approches théoriques de la communication confrontées à l'individuation

Les 38 articles scientifiques reposent, selon nous, sur cinq approches théoriques : analyse critique du discours, analyse de l'activité, théorie de la structuration, théorie de la traduction et communication constitutive de l'organisation. Ces approches théoriques peuvent être rattachées à trois paradigmes : critique pour l'analyse critique du discours, interprétatif pour l'analyse de l'activité et constructionniste pour la théorie de la structuration, la théorie de la traduction et la communication constitutive de l'organisation.

En guise de discussion, nous nous sommes demandé comment chacune de ces théories, étant donné son ancrage paradigmatique, peut-elle contribuer à une meilleure compréhension de la constitution de l'individu. L'idée est bien de mettre en évidence les glissements à opérer potentiellement pour y intégrer cette troisième stratégie de l'individuation.

\subsubsection{Paradigme critique : l'analyse critique du discours}

L'analyse critique questionne les logiques de domination, d'assujettissement, de distribution du pouvoir telles que pensées par Foucault :

« Du moment qu'à travers les pratiques il s'agit d'étudier les différents modes d'objectivation du sujet, on comprend la part importante que doit occuper l'analyse des relations de pouvoir. Mais encore faut-il bien définir ce que peut et ce que veut être une pareille analyse. II ne s'agit pas d'interroger le 'pouvoir' sur son origine, ses principes ou ses limites légitimes, mais d'étudier les procédés et techniques qui sont utilisés 
dans différents contextes institutionnels pour agir sur le comportement des individus pris isolément ou en groupe ; pour former, diriger, modifier leur manière de se conduire, pour imposer des fins à leur inaction ou l'inscrire dans des stratégies d'ensemble. Ces relations de pouvoir caractérisent la manière dont les hommes sont 'gouvernés' les uns par les autres; et leur analyse montre comment, à travers certaines formes de 'gouvernement' [...], est objectivé le sujet » (Foucault, 1994, 635).

Même si dans l'approche critique la condition du sujet en organisation est centrale, nous ne considérons pas pour autant que l'on donne une place prépondérante à sa capacité de réflexion comme point initial de la recherche. Le numéro 28 consacré à Foucault tend à montrer comment de nouvelles formes de gouvernementalité s'imposent aux acteurs. La faible place donnée à l'empirie dans le dossier nous laisse sans réponse quant à l'intention des acteurs. Les approches critiques foucaldiennes (celles basées sur les premiers travaux de Foucault), de par leur positionnement épistémologique structuraliste, laissent, par ailleurs, peu de place aux acteurs, à leurs récits et à leurs singularités. Pour autant, des ouvertures existent dans ces travaux. Pour Heller, notamment, les travaux critiques doivent interroger le management comme une « instrumentalisation du rapport à soi à des fins de docilité/utilité » (Heller, 2005a, § 40). L'analyse critique des discours se concentrera, dès lors, sur la façon dont la gouvernementalité s'appuie «sur un ensemble de discours très présents dans la société sur le soi, sur les rapports à soi et à l'autre, sur la façon de se transformer pour accéder à un sentiment de bien-être, de bonheur, à une certaine connaissance de soi. Elle s'étaye donc sur ce qu'on pourrait appeler une idéologie du soi, mais aussi des techniques de soi que l'organisation productive récupère, adapte à son contexte et à son profit »(Heller, 2005a, § 44).

\subsubsection{Paradigme interprétativiste : I'analyse de l'activité}

L'analyse de l'activité en SIC se concentre essentiellement sur les activités dites d'inscription :

"Les écrits dits de travail font partie de ces inscriptions qui orientent l'action, la stabilisent et contribuent à en articuler les différentes composantes. C'est à la fois une ressource et un moyen mobilisé dans l'activité de travail en ce sens qu'il la formate, la structure alors même qu'il est un produit de cette même activité de travail. Les acteurs ont besoin de ces écrits pour exécuter leur tâche mais également pour faire savoir, pour passer le relais » (Mayère et Roux, 2009, § 4).

L'intérêt pour l'intention, l'explicitation de l'agir, de la réflexion sur le soi dans une approche diachronique se retrouvent particulièrement dans les travaux de l'analyse de l'activité équipée en milieu hospitalier (Jolivet, 2010). Le fait que ces travaux portent en particulier sur des métiers vocationnels (dans le secteur des soins de santé ou de la justice), de par le choix de carrière que ces pratiques professionnelles impliquent, offrent des parcours de vies très 
riches pour analyser les épreuves auxquelles les acteurs sont soumis. Notamment, l'épreuve du travail est centrale dans les phénomènes observés. L'analyse de l'activité en sciences de la communication doit être attentive aux aspects communicationnels et informationnels du travail (Licoppe et al., 2010). Pour Pierre Delcambre $(2009, \S 3)$ :

"L'activité de l'acteur (fil n 1) n'est pas seulement aux prises avec le système d'activité socialement organisé - dispositifs, organisation du travail, 'délégation' ou 'assistance' par les machines et l'outillage (fil $\left.n^{\circ} 2\right)$ : cette activité met en jeu sur le lieu du travail des gestes, un 'faire' installé ou dérouté par le travail contraint généré par les systèmes d'information, mais aussi par les différents 'formats' du travail langagier. Mais encore, la 'communication' au travail ne s'origine pas que des échanges nécessaires à l'activité 'collective' (ajustement, coordination...), phénomènes bien pris en compte par nos collègues sociologues; elle s'origine aussi du dialogue avec soi-même pour penser sa propre tâche et résoudre les problèmes de l'effectuation du travail, donc de la sollicitation de discours, d'informations, de documents qui actualisent le sens de ce que l'on fait et permettent de suivre le cours d'action ».

Ces caractéristiques (la place donnée à l'individu, à son récit objectif et subjectif, à la mise à l'épreuve du travail, à l'apprentissage qui en découle) font, semble-t-il, de l'analyse de l'activité une approche pouvant adopter l'agenda de l'individuation afin d'étudier les mutations actuelles du travail.

3.2.3. Paradigme constructionniste : théorie de la structuration, théorie de la traduction et la communication dite constitutive de l'organisation

Le paradigme constructionniste recouvre, dans les dossiers thématiques traités, trois théories : la théorie de la structuration, la théorie de la traduction et la communication constitutive de l'organisation.

Le cœur de la théorie de la structuration est le questionnement sur l'évolution des pratiques sociales, des rapports sociaux entre les acteurs évoluant dans un système social fait de règles et de ressources. Reposant sur la dualité de la structure développée par Giddens (1984), ces travaux tentent de comprendre dans quelle mesure la structuration d'une activité permet - ou non - aux acteurs d'avoir une autonomie de "bricolage " des TIC qui semble nécessaire à l'appropriation du dispositif sociotechnique » (Comtet, 2009, § 2). La grande majorité des articles analysés et qui reposent sur cette approche questionne l'usage et l'appropriation d'un dispositif de communication (Douyère, 2009) dans une pratique sociale en contexte organisationnel.

Ces travaux, parce qu'ils reposent grandement sur les entretiens, donnent également une place importante à la parole des individus, à leur réflexivité et à leurs intentions. Même si ces recherches s'inscrivent dans une démarche diachronique, elles ne placent pas leur réflexion sur la mutation des pratiques 
sociales dans un temps long.

En déclin, l'approche structurationniste de l'usage et de l'appropriation des dispositifs de communication a cédé progressivement sa place au courant dit de la socio-matérialité qui inclut les actants non-humains à sa réflexion. Ces travaux reposent sur la théorie de la traduction (Akrich et al., 2006). Dans cette théorie, l'intérêt porte sur le caractère distribué de la performation dans l'agencement du réseau d'acteurs. Les scripts désignent alors :

"L'agrégat de mots d'ordres et de schémas stratégiques combinés, mis en visibilité et circulant dans les référentiels d'actions, les injonctions répétées, auxquels sont confrontées les organisations, au travers de leurs traductions normatives et coercitives (palmarès des meilleurs intranets, idéaux-types, livres-blancs, solutions technologiques standardisées, répertoires de réponses, prescriptions dogmatiques etc.). Ils sont un des actants organisateurs des champs de visibilité et des régimes d'énoncés qui vont caractériser la politique organisationnelle concernée et le champ de prescriptions stratégiques qui s'y rattache " (Carmes, 2008, 3).

Dans ces deux théories, un glissement vers une stratégie de l'individuation impliquerait de penser les dispositifs de communication non pas comme élément perturbateur du système social mais dans une perspective historique ou sociétale de grande ampleur au sens où les épreuves sont définies par Martuccelli. Les récits de la mise en œuvre ou de l'appropriation des dispositifs devenant un terrain propice à l'analyse de plus grandes épreuves comme celle de la transformation du travail se manifestant par la mise en œuvre répétée de dispositifs numériques.

La troisième approche théorique de ce paradigme est la communication constitutive de l'organisation qui considère que la communication organisationnelle n'exprime pas seulement la réalité sociale ou organisationnelle, mais elle la crée (Searle, 1995). L'analyse est ici centrée sur ce que produit la communication qu'elle soit conversationnelle ou textuelle. Certains auteurs vont se questionner sur ce qui est produit dans l'interaction. C'est le cas de Vasquez et ses collègues $(2013, \S 2)$ qui envisagent le branding " comme un processus interactionnel et communicationnel de re-présentations où des pratiques spécifiques de production, de consommation et de distribution des marques entre des agents ou acteurs organisationnels puis des consommateurs ". D'autres vont s'intéresser à la nature textuelle de l'organisation (Robichaud et Benoit-Barné, 2010) ou à l'agentivité du texte (normatif ou prescriptif) en organisation (Chantraine, 2001 ; Fauré, 2010 ; Piponnier, 2010 ; Lambotte et Scieur, 2013). Pour reprendre les mots de Bertrand Fauré (2010, § 5-7) :

«Cette agentivité des textes peut être expliquée à l'aide de deux principaux mécanismes ou propriétés : la méta-textualité et la performativité. La méta-textualité désigne le fait qu'un texte s'inscrive - ou non - dans 
un réseau textuel plus vaste qui lui donne sens et légitimité. Par exemple, une procédure ne peut être comprise et appliquée que si elle fait référence à d'autres textes normatifs qui la définissent comme telle et lui donnent autorité. L'agencement de ces différents niveaux de textes et les méta-conversations (Robichaud et al., 2004) qu'ils instaurent crée leur force. Appliquée à l'influence normative des textes, la notion de performativité initialement élaborée par les philosophes du langage (Austin, 1962 ; Searle, 1972) permet de comprendre comment - et avec quels effets - les textes sont parlés, cités, convoqués ainsi que comment en retour ils font parler et agir ceux qui les énoncent " (Denis, 2006 ; Fauré et Gramaccia, 2006).

Les travaux dans cette approche, en particulier ceux se réclamant de l'école de Montréal, sont critiqués pour leur absence de questionnement sur la notion de pouvoir ou encore pour leur approche centrée sur la communication évacuant la question des individus et leur intentionnalité. Ces travaux se concentrent sur ce qui est produit dans l'interaction ou encore sur la performativité d'agents non-humains comme les textes. Des réflexions récentes ont amenés plusieurs chercheurs de cette veine à s'intéresser à la constitution de la réalité et de ces différents modes d'existences (Latour, 2012). Il est dès lors plausible que l'individu devienne le centre du questionnement de ce qui est constitué dans la communication. En effet, l'analyse fine des échanges et la proximité avec les individus sont des caractéristiques centrales des approches des CCO dans lesquelles le travail ethnographique (Grosjean et Bonneville, 2009 ; Brummans, 2007) prend une place prépondérante.

Néanmoins les démarches diachroniques y restent limitées. Partant du principe d'endogénéité des interactions, ces travaux privilégient l'analyse conversationnelle plutôt que l'analyse sur un temps long. Des travaux récents cherchent à inscrire la réflexion sur la constitution des organisations dans un temps long par la mise en récit ou l'imbrication des événements de communication dans une perspective longitudinale (Martin-Scholz, 2017). Ces travaux offrent un bagage conceptuel propice à l'analyse constitutive dans une perspective diachronique qui pourrait être adaptée à l'individu.

\section{3.}

\section{Vers une mise en récit des épreuves sociales en contexte organisationnel}

En guise d'ouverture vers de nouvelles perspectives, nous discutons de deux éléments essentiels de l'agenda de recherche de l'individuation : les épreuves et leur mise en récit par le prisme de l'individu.

Est-il possible de replacer les phénomènes organisationnels dans une perspective plus large d'épreuves sociales ? Si oui, quelles sont-elles ? Les nou- 
velles formes et normes du management et de gouvernementalité, la création et la mise à disposition des dispositifs numériques ou techniques à des fins de contrôles, d'amélioration des performances sont autant de manifestation de l'épreuve du travail comme épreuve centrale mais qui, on le perçoit, font surgir d'autres mises à l'épreuve comme celle du rapport au collectif, à soi mais également à la famille. Par exemple, dans son article sur les start-ups, Estienne (2005) montre bien comment les frontières entre vies privée et professionnelles s'estompent permettant le développement du travail hors du cadre spatio-temporel du travail envahissant des sphères qui sont initialement prévues pour la vie privée.

Ces manifestations concrètes des épreuves sont donc bien présentes dans notre corpus d'articles et elles sont remises en perspective par rapport à d'autres mécanismes sociaux (e.a., la managérialisation des organisations, l'augmentation de la place des technologies dans le travail, etc.) qui produisent ces épreuves. Pour Martuccelli (2007, § 32), « il s'agit de saisir les structures historiques au travers des situations individuelles, l'analyse s'emparant des coercitions à partir des trajectoires personnelles. Cependant, pour en rendre compte autrement que sous l'unique forme d'expériences individuelles, l'analyse doit également cerner les différents mécanismes sociaux qui les produisent (et ceci de la manière la plus concrète possible). La prise de vue panoramique doit ainsi constamment aller de pair avec le gros plan, afin de cerner les multiples diffractions des processus à l'œuvre ". Cependant ces manifestations ne sont pas replacées dans une perspective sociohistorique par le biais des récits. Seule exception, dans leur article, Olivia Foli et Marlène Dulaurans (2013) exposent à travers de deux récits de chercheures sous contrat CIFRE les difficultés à appréhender l'ambiguïté du double statut ambigu chercheur-employé lorsque soumis aux contraintes organisationnelles.

Si cette troisième voie d'étude de l'individu est empruntée, les travaux récents montrent que la narratologie met à disposition un certain nombre d'outils conceptuels qui permettraient aux chercheurs en SIC de se démarquer des sociologues de par la rigueur scientifique du regard posé sur récit des individus ; élément central du dispositif de Martuccelli. À titre d'exemple, les chercheurs en SIC se sont questionnés sur la nature même du récit de vie ou de pratique (Demazière, 2003 ; de la Broise, 2008). De même l'approche narrative des organisations développée par Barbara Czarniawska (2004), notamment, permet une analyse fine des récits mettant en lumière l'entrelacement, les tensions, les ambiguïtés auxquelles sont soumises les individus. Ces outils conceptuels peuvent contribuer à une compréhension rigoureuse de l'individu aux prises avec les épreuves de la vie en organisation dans une perspective sociohistorique plus longue que celle habituellement de mise dans les travaux en communication organisationnelle. 
Bibliographie

Akrich M., Callon M., Latour B. (2006). Sociologie de la traduction : Textes fondateurs, Paris, Presses de l'École des Mines.

Bouillon J.-L. (2005). «Autonomie professionnelle et rationalisations cognitives : les paradoxes dissimulés des organisations post-disciplinaires ». In Études de communication, $n^{\circ} 28, p .91-105$. Disponible sur https://doi.org/10.4000/edc.286 (page consultée le 14 février 2018).

Brummans B. H. J. M. (2007). "Travels of a Buddhist Mind". In Qualitative Inquiry, vol. $13, \mathrm{n}^{\circ} 8$, p. 1221-1226. Disponible sur https:// doi.org/10.1177/1077800407308225 (page consultée le 14 février 2018).

Carmes M. (2008). « L'organisation peut-elle s'inventer par les scripts? : Performations et actualisations dans les agencements intranet ". In Actes du colloque L'organisation peut-elle s'inventer par les scripts ?, Université de Nice Sophia Antipolis, France, p. 266-274. Disponible sur https://hal.archives-ouvertes. $\mathrm{fr} /$ hal-00411233/document (page consultée le 14 février 2018).

Carmes M. (2009). « Les activités de contribution dans la politique intranet d'un Conseil Général : de I'injonction à une sémio-politique de la 'transversalité' en organisation ". In Études de communication, $n^{\circ} 33$, p. 19-40. Disponible sur https:// doi.org/10.4000/edc.1105 (page consultée le 14 février 2018).

Chantraine O. (2001). "Le 'carnet de santé 1996' : autopsie d'un support de communication mort-né ". In Études de communication, $\mathrm{n}^{\circ} 23$, p. 51-68. Disponible sur https:// doi.org/10.4000/edc.1138 (page consultée le 14 février 2018).

Comtet I. (2009). « Entre usage professionnel des TIC et structure organisationnelle : la capacité au bricolage comme compétence adaptative ". In Études de communication, $n^{\circ} 33$, p. 119-134. Disponible sur https://doi.org/10.4000/edc.1079 (page consultée le 14 février 2018).

Cooren F. (2010). "Comment les textes écrivent l'organisation.

Figures, ventriloquie et incarnation ». In Études de communication, $n^{\circ} 34$, p. 23-40. Disponible sur https:// doi.org/10.4000/edc.1891 (page consultée le 14 février 2018).

Czarniawska B. (2004). "On Time, Space, and Action Nets". In Organization, vol. 11, $n^{\circ} 6$, p. 773791. Disponible sur https://doi. org/10.1177/1350508404047251 (page consultée le 14 février 2018).

Delcambre P. (2009). « L'activité d'évaluation et les systèmes d'information. L'évaluation est aussi un travail : langagier, assisté, organisé ». In Études de communication, $\mathrm{n}^{\circ} 33$, p. 79-100. Disponible sur https:// doi.org/10.4000/edc.1028 (page consultée le 14 février 2018). 
Demazière D. (2003). « Matériaux qualitatifs et perspective longitudinale. La temporalité des parcours professionnels saisis par les entretiens biographiques ". In Les données longitudinales dans l'analyse du marché du travail, $10^{\text {es }}$ Journées d'études Céreq-Lasmas-IdL, Caen, 21, 22 et 23 mai 2003, p. 75-89.

Douyère D. (2009). « Activité et contrôle de l'information sur un intranet d'entreprise : censure et régulation du discours sur le stress au travail ». In Études de communication, $n^{\circ} 33$, p. 41-56. Disponible sur https://doi.org/10.4000/edc.1037 (page consultée le 14 février 2018).

Estienne Y. (2005). «La mobilisation des (net)travailleurs de la 'Nouvelle économie' : gouvernement des hommes et contrainte d'autonomie ". In Études de communication, $\mathrm{n}^{\circ} 28$, p. 15-30. Disponible sur https:// doi.org/10.4000/edc.77 (page consultée le 14 février 2018).

Fauré B. (2010). « L'agentivité textuelle entre métatextualité et performativité. Le cas des Operguid dans l'industrie pétrochimique ". In Études de communication, $\mathrm{n}^{\circ} 34$, p. 61-74. Disponible sur https:// doi.org/10.4000/edc.1660 (page consultée le 14 février 2018).

Floris B., Ledun M. (2005). « Le marketing, technologie politique et forme symbolique du contrôle social ». In Études de communication, $n^{\circ} 28$, p. 125-140. Disponible sur https://doi.org/10.4000/edc.303 (page consultée le 14 février 2018).
Foli O., Dulaurans M. (2013). « Tenir le cap épistémologique en thèse Cifre. Ajustements nécessaires et connaissances produites en contexte ». In Études de communication, $\mathrm{n}^{\circ} 40$, p. 59-76. Disponible sur https:// doi.org/10.4000/edc.5118 (page consultée le 14 février 2018).

Foucault M. (1994). Dits et écrits (1954-1988), tome II : 1976-1988, Paris, Gallimard.

Giddens A. (1984). The constitution of society : outline of the theory of structuration, Cambridge, Polity.

Giroux N., Demers C. (1998).

"Communication organisationnelle et stratégie ". In Management International, vol. 2, $\mathrm{n}^{\circ}$ 2, p. 17-32.

Gléonnec M. (2009). « Le lien social dans les systèmes d'information : le cas des services de santé au travail ". In Études de communication, $\mathrm{n}^{\circ} 33$, p. 135-152. Disponible sur https:// doi.org/10.4000/edc.1070 (page consultée le 14 février 2018).

Grosjean S., Bonneville L. (2009). "Saisir le processus de remémoration organisationnelle des actants humains et non humains au cœur du processus ". In Revue d'anthropologie des connaissances, vol. $3, \mathrm{n}^{\circ} 2$, p. 317-347. Disponible sur https:// doi.org/10.3917/rac.007.0317 (page consultée le 14 février 2018).

Guyot B. (2009a). «Introduction ». In Études de communication, $n^{\circ} 33$, p. 9-18. Disponible sur https:// doi.org/10.4000/edc.1069 (page consultée le 19 février 2018). 
Guyot B. (2009b). «Se mouvoir au sein du monde de l'information : comment les personnes parlent de leur activité d'information ". In Études de communication, $\mathrm{n}^{\circ} 33$, p. 101-118. Disponible sur https:// doi.org/10.4000/edc.1052 (page consultée le 19 février 2018).

Heller T. (2005a). « De l'anatomopolitique à la psychopolitique ». In Études de communication, $\mathrm{n}^{\circ} 28$, p. 59-75. Disponible sur https:// doi.org/10.4000/edc.265 (page consultée sur le 19 février 2018).

Heller T. (2005b). "Introduction ». In Études de communication, $\mathrm{n}^{\circ} 28$, p. 7-30. Disponible sur https:// doi.org/10.4000/edc.265 (page consultée sur le 19 février 2018).

Jolivet A. (2010). « Voir les normes comme un travail de sens : le texte comme agencement de narrations ». In Études de communication, $\mathrm{n}^{\circ} 34$, p. 75-92. Disponible sur https:// doi.org/10.4000/edc.1755 (page consultée le 14 février 2018).

Kogan A.-F. (2009). " La mise en jeu du corps dans l'activité de téléphonie ". In Études de communication, $n^{\circ} 33$, p. 171-186. Disponible sur https://doi.org/10.4000/edc.1059 (page consultée le 14 février 2018).

La Broise P. de (2008). « L'entretien biographique : plus qu'une méthode, une méthodologie pour la recherche en communication organisationnelle ». In A. Bouzon et V. Meyer (dir.), La communication des organisations : entre recherche et action, Paris, L'Harmattan, (p. 105-115).
La Broise P. de, Grosjean S. (2010).

"Introduction ». In Études de communication, $n^{\circ} 34$, p. 9-22. Disponible sur http://journals. openedition.org/edc/1752 (page consultée le 19 février 2018).

Lamarche T. (2001). "Service public : nouvelle rationalité des acteurs ou nouveau marché ? ». In Études de communication, $\mathrm{n}^{\circ} 23$, p. 69-88. Disponible sur https:// doi.org/10.4000/edc.1139 (page consultée le 14 février 2018).

Lambotte F., Scieur P. (2013). « Le statut du rapport dans l'audit organisationnel : de sa légitimation par l'écriture à son action comme agent non humain $»$. In Études de communication, $\mathrm{n}^{\circ} 40$, p. 111-128. Disponible sur https:// doi.org/10.4000/edc.5154 (page consultée le 14 février 2018).

Latour B. (2012). Enquête sur les modes d'existence : une anthropologie des modernes, Paris, La Découverte.

Le Moënne C. (2013). «Entre formes et normes. Un champ de recherches fécond pour les SIC ». In Revue française des sciences de l'information et de la communication, $\mathrm{n}^{\circ} 2$. Disponible sur https:// doi.org/10.4000/rfsic.365 (page consultée le 14 février 2018).

Licoppe C., Proulx S., Cudicio R. (2010). " L'émergence d'un nouveau genre communicationnel dans les organisations fortement connectées : les 'questions rapides' par messagerie instantanée ». In Études de communication, $\mathrm{n}^{\circ} 34$, 
p. 93-108. Disponible sur https:// doi.org/10.4000/edc.1661 (page consultée le 14 février 2018).

Martin-Scholz A. (2017). Communiquer et organiser en échafaudant un big data manuel. Le cas d'un projet de formation en matière d'aménagement du territoire, Thèse de doctorat, Université de Toulouse - Jean Jaurès, Toulouse.

Martuccelli D. (2005). "Les trois voies de l'individu sociologique ". In EspacesTemps.net. Disponible sur https://www.espacestemps. net/articles/trois-voies-individu-sociologique/ (page consultée le 14 février 2018).

Martuccelli D. (2007). « Les épreuves de l'individu dans la globalisation ». In Recherches sociologiques et anthropologiques, vol. 38, $n^{\circ} 1$, p. 13-32. Disponible sur https:// doi.org/10.4000/rsa.508 (page consultée le 14 février 2018).

Martuccelli D. (2009). «Qu'est-ce qu'une sociologie de l'individu moderne ? Pour quoi, pour qui, comment? ». In Sociologie et sociétés, vol. 41, $n^{\circ} 1$, p. 15-33. Disponible sur https://doi.org/10.7202/037905ar (page consultée le 14 février 2018).

Mayere A., Roux A. (2009). "Écritures individuelles et collectives. Déclarer, ne pas déclarer, savoir dire et savoir jouer avec le dispositif ». In Études de communication, $\mathrm{n}^{\circ} 33$, p. 57-78. Disponible sur https:// doi.org/10.4000/edc.1162 (page consultée le 14 février 2018).
Morillon L., Bouzon A., Lee C. (2013). « Épistémologies, théories et pratiques professionnelles en communication des organisations ". In Études de communication, $n^{\circ} 40$, p. 9-26. Disponible sur https:// doi.org/10.4000/edc.5038 (page consultée le 14 février 2018).

Noyer J. (2001). " L'usager' télé-visé : TGV bloqués, caténaires gelées et médiatisation de l'information en situation perturbée ». In Études de communication, $\mathrm{n}^{\circ} 23$, p. 105-128. Disponible sur https:// doi.org/10.4000/edc.1141 (page consultée le 14 février 2018).

Noyer J., Chantraine O. (2001). «Introduction ». In Études de communication, $n^{\circ} 23$, p. 7-10. Disponible sur https://doi. org/10.4000/edc.1133 (page consultée le 19 février 2018).

Petitet V. (2005). « La gouvernementalité managériale ». In Études de communication, $\mathrm{n}^{\circ} 28$, p. 31-44. Disponible sur https:// doi.org/10.4000/edc.246 (page consultée le 14 février 2018).

Piponnier A. (2010). « Observer pour gouverner : information, prescription et médiation dans les observatoires numériques territoriaux ". In Études de communication, $\mathrm{n}^{\circ} 34$, p. 109-126. Disponible sur https://doi.org/10.4000/edc.1767 (page consultée le 14 février 2018).

Raoul B. (2001). «Technologies de l'information et de la communication et modernisation des services publics. Quelques remarques et 
repères pour une exploration cri-

tique ». In Études de communication, $\mathrm{n}^{\circ} 23$, p. 11-30. Disponible sur https:// doi.org/10.4000/edc.1135 (page consultée le 14 février 2018).

Robichaud D., Benoit-Barné C. (2010). «L'épreuve de la conversation : comment se négocie la mise en œuvre des normes dans l'écriture d'un texte organisationnel ». In Études de communication, $\mathrm{n}^{\circ} 34$, p. 41-60. Disponible sur https://doi.org/10.4000/edc.1802 (page consultée le 14 février 2018).

Salançon A. (2009). «Innovation informationnelle et changements organisationnels : l'exemple de la traçabilité agroalimentaire informatisée ". In Études de communication, $\mathrm{n}^{\circ} 33$, p. 153-169.

Searle J. R. (1995). The construction of social reality, New York, Simon \& Schuster.

Strauss A. L., Corbin J. (1998). Basics of Qualitative Research: Techniques and Procedures for Developing Ground Theory, Thousand Oaks, Sage Publications.

Vásquez C., Cordelier B., Sergi V. (2013). " Pratiques de branding en contexte universitaire. Une approche processuelle de la marque ». In Études de communication, $\mathrm{n}^{\circ} 40$, p. 93-110. Disponible sur https:// doi.org/10.4000/edc.5143 (page consultée le 14 février 2018).

\section{Références Études de communication}

Études de communication, Dossier Services aux publics : question de communication et de management, $\mathrm{n}^{\circ} 23,2001$.

Études de communication, Dossier Organisation, dispositif, sujet, $\mathrm{n}^{\circ} 28,2005$.

Études de communication, Dossier L'activité aux prises avec des systèmes ou dispositifs d'information, $n^{\circ} 33,2009$.

Études de communication, Dossier Normes et écriture de l'organisation, n 34, 2010.

Études de communication, Dossier Épistémologies, théories et pratiques professionnelles en communication des organisations, $n^{\circ}$ 40, 2013. 


\section{Annexe 1}

Ces chiffres (voir tableau 1) sont à prendre avec précaution mais méritent que l'on s'y attarde vu la qualité des réflexions scientifiques proposées dans chacun des numéros. L'ensemble de ces informations fournit une indication sur l'ouverture et la portée des travaux dans le monde francophone de la recherche en sciences de l'information et de la communication (SIC). Les métadonnées des cinq numéros sont reprises dans le tableau ci-dessous.

\begin{tabular}{|c|c|c|c|c|}
\hline $\begin{array}{l}\text { Numéro / } \\
\text { Année }\end{array}$ & Titre & $\begin{array}{l}\text { Éditeur(s). } \\
\text { trice(s) }\end{array}$ & Résumé & $\begin{array}{c}\text { Nombre } \\
\text { d'articles } \\
\text { / Index de } \\
\text { citations } \\
\text { Google }\end{array}$ \\
\hline $23 / 2001$ & $\begin{array}{l}\text { Services aux } \\
\text { publics: } \\
\text { question } \\
\text { de commu- } \\
\text { nication } \\
\text { et de } \\
\text { management }\end{array}$ & $\begin{array}{c}\text { Jacques } \\
\text { Noyer et } \\
\text { Olivier } \\
\text { Chantraine }\end{array}$ & $\begin{array}{l}\text { Qu'en est-il des mutations qu'ont } \\
\text { à affronter les organismes dits de } \\
\text { " services aux publics " quelle } \\
\text { place y prennent la communication } \\
\text { et les professionnels de ce } \\
\text { secteur ? Que recouvre, dans un } \\
\text { tel contexte, le " management de } \\
\text { la communication "? Quel accom- } \\
\text { pagnement, quelle construction du } \\
\text { changement les nouvelles pratiques } \\
\text { organisationnelles induisent-elles en } \\
\text { matière de " service"? }\end{array}$ & $7 / 13$ \\
\hline $28 / 2005$ & $\begin{array}{l}\text { Organisation, } \\
\text { dispositif, } \\
\text { sujet }\end{array}$ & $\begin{array}{c}\text { Thomas } \\
\text { Heller }\end{array}$ & $\begin{array}{l}\text { Les transformations qui touchent } \\
\text { les organisations productives } \\
\text { depuis plus de vingt ans mettent- } \\
\text { elles un terme aux dispositifs } \\
\text { disciplinaires, dans leur contenu et } \\
\text { dans leur logique, tels qu'ils ont été } \\
\text { analysés par Michel Foucault ? Y } \\
\text { a-t-il une spécificité des dispositifs } \\
\text { actuels liée à ces changements? } \\
\text { Et qu'est-ce que la rencontre } \\
\text { entre désir et gouvernement, } \\
\text { qui semble caractériser le mana- } \\
\text { gement moderne, nous dit de } \\
\text { la place qu'occupe le sujet dans } \\
\text { l'organisation, c'est-à-dire en tant } \\
\text { qu'il n'est plus constitué par les } \\
\text { disciplines?... }\end{array}$ & $10 / 83$ \\
\hline
\end{tabular}




\begin{tabular}{|c|c|c|c|c|}
\hline $33 / 2009$ & $\begin{array}{c}\text { L'activité } \\
\text { aux prises } \\
\text { avec des } \\
\text { systèmes } \\
\text { ou } \\
\text { dispositifs } \\
\text { d'information }\end{array}$ & Brigitte Guyot & $\begin{array}{l}\text { Études de communication élargit } \\
\text { considérablement le terme d'usage } \\
\text { en l'entendant, dans cette livraison, } \\
\text { de façon large comme étant la "vie } \\
\text { autour de l'information ". Seront } \\
\text { traitées les manières dont chacun } \\
\text { s'organise pour obtenir, produire et } \\
\text { gérer l'information dont il a besoin } \\
\text { pour travailler, s'aidant en cela } \\
\text { d'outils et de systèmes, ou s'en } \\
\text { créant de nouveaux... }\end{array}$ & $10 / 43$ \\
\hline $34 / 2010$ & $\begin{array}{c}\text { Normes } \\
\text { et } \\
\text { écriture } \\
\text { de } \\
\text { l'organisation }\end{array}$ & $\begin{array}{l}\text { Patrice de } \\
\text { la Broise } \\
\text { et Sylvie } \\
\text { Grosjean }\end{array}$ & $\begin{array}{l}\text { La question des normes constitue un } \\
\text { objet éminemment heuristique pour } \\
\text { la recherche en sciences humaines } \\
\text { et sociales, et plus particulièrement } \\
\text { en sciences de l'information et de } \\
\text { la communication, sachant que } \\
\text { l'activité sociale est tout à la fois } \\
\text { tributaire et productrice de ces } \\
\text { normes. De nombreux chercheurs, } \\
\text { tant français que nord-américains, } \\
\text { ont étudié le rôle de diverses formes } \\
\text { de textes, d'écritures dans les } \\
\text { organisations... }\end{array}$ & 9 / 63 \\
\hline $40 / 2013$ & $\begin{array}{l}\text { Epistémo- } \\
\text { logies, } \\
\text { théories } \\
\text { et pratiques } \\
\text { profession- } \\
\text { nelles } \\
\text { en commu- } \\
\text { nication des } \\
\text { organisations }\end{array}$ & \begin{tabular}{|c|} 
Laurent \\
Morillon, \\
Arlette \\
Bouzon et \\
Carolyne Lee
\end{tabular} & $\begin{array}{l}\text { Quels rapports les pratiques profes- } \\
\text { sionnelles entretiennent-elles avec } \\
\text { les théories dans le domaine de la } \\
\text { communication des organisations? }\end{array}$ & $7 / 19$ \\
\hline
\end{tabular}

Tableau 1 : métadonnées des cinq numéros thématiques analysés 
